\title{
Agon HAMzA (ed.), Althusser and Theology. Religion, Politics and Philosophy
}

Leyde-Boston, Brill, coll. « Historical materialism », 2016, 210 p.

\section{Cornelius Crowley}

\section{(2) OpenEdition}

Journals

Édition électronique

URL : https://journals.openedition.org/assr/44924

DOI : $10.4000 /$ assr.44924

ISSN : 1777-5825

Éditeur

Éditions de l'EHESS

Édition imprimée

Date de publication : 1 décembre 2018

Pagination : 298-302

ISSN : 0335-5985

\section{Référence électronique}

Cornelius Crowley, "Agon hamza (ed.), Althusser and Theology. Religion, Politics and Philosophy », Archives de sciences sociales des religions [En ligne], 184 I octobre-décembre 2018, mis en ligne le 01 décembre 2018, consulté le 15 janvier 2022. URL : http://journals.openedition.org/assr/44924 ; DOI : https://doi.org/10.4000/assr.44924

Ce document a été généré automatiquement le 15 janvier 2022.

(c) Archives de sciences sociales des religions 


\title{
Agon HAmza (ed.), Althusser and Theology. Religion, Politics and Philosophy
}

Leyde-Boston, Brill, coll. « Historical materialism », 2016, 210 p.

\author{
Cornelius Crowley
}

\section{RÉFÉRENCE}

Agon HAmzA (ed.), Althusser and Theology. Religion, Politics and Philosophy, Leyde-

Boston, Brill, coll. « Historical materialism », 2016, 210 p.

1 Le volume offre douze perspectives sur le «projet philosophique» (p.1) d'Althusser. Elles ont en commun le point d'entrée qu'est son articulation avec «la théologie». Agon Hamza, dans son introduction - «Althusser's Christian Marxism» (p. 1-6) -, cite Balibar (1993), qui pointe "l'inscription conjoncturelle» du projet philosophique d'Althusser : "For these reasons, his work is "heavily dependent on a certain context" because it has an "intervening" character and "the fate of any intervention is to wither away in its own effects" ". Les contributions portent prioritairement sur les écrits d'Althusser de la période 1945-1951, qui dans le volume 1 des Écrits philosophiques et politiques (1994) vont de «L'internationale des bons sentiments » (1946) à «Lettre à Jean Lacroix » (1950-51) et «Sur l'obscénité conjugale» (1951) et sont regroupés sous le titre général «Louis Althusser avant Althusser ».

2 Le contexte politique et moral de l'après-guerre, tel qu'il se réfléchit en ce moment du parcours philosophique d'Althusser, fait l'objet de l'article de Geoff Pfeifer, «Between Hegel and Marx: History and Theology in the Early Althusser " (p. 89-100). La dimension conjoncturelle sera de nouveau interrogée dans l'article de Jana Tsoneva, «From the "International of Decent Feelings" to the International of Decent Actions: Althusser's Relevance for the Environment Conjuncture of Late Capitalism » (p. 18-181). Chez Tsoneva, la lecture du texte d'Althusser en relation avec le contexte de sa rédaction (1946), à savoir le 
climat de désarroi de l'après-guerre, démontre que les analyses sont indiscutablement réflexives et d'une visée critique en leur contexte d'intervention. Elles portent sur une conjoncture européenne précise, sur les possibles lectures religieuses ou théologiques de celle-ci. Le texte de 1946 a ainsi pour effet l'évaluation critique des discours herméneutiques et apologétiques suscités par ce désarroi. Ces discours visent une compréhension de la conjoncture en faisant de ses traits les plus marquants, « deux ans après la plus atroce des guerres, sur la terre couverte de paix et de ruines » (Althusser, 1994, p. 35-36), les motifs de sa qualification expressive, selon des procédures dont Althusser pointe les complaisances.

Or «L'internationale des bons sentiments " peut désormais nous interpeller dans un autre climat, celui d'une détresse écologique sensible en ce moment du capitalisme tardif. Ainsi, le critère de l'inscription conjoncturelle du projet d'Althusser garde-t-il sa pertinence comme clé d'entrée dans le parcours philosophique. Il se vérifie à la lecture des perspectives proposées dans le présent volume. S'agissant de «L'internationale des bons sentiments ", l'inscription contextuelle initiale, doublée d'une réinscription ultérieure dans une conjoncture autre, atteste l'aptitude du projet althussérien à pratiquer, dans plusieurs contextes distincts, la critique des convenances idéologiques qui, en 1946 ou de nos jours, concourent à la production d'une figure expressive de la conjoncture, ainsi dotée de traits à la fois cohérents et convenables. La traduction en anglais du texte de 1946 s'intitule "The International of Decent Feelings", decency nommant un ordre ajusté à l'idée régulatrice de ce qui est convenable. Dans son contexte d'origine ou dans le contexte de notre présent, «L'internationale des bons sentiments » peut être hissée comme bannière inclusive permettant de penser un destin commun, vécu sous la menace de la bombe atomique ou d'un effondrement climatique. Dans les deux cas, l'idée d'un destin commun surplombé d'une menace commune permettrait la neutralisation des conflits de classe ou de territoires, dans une conjoncture de guerre froide ou dans celle du capitalisme tardif. La double contextualisation pratiquée par Tsoneva valide l'hypothèse que le projet philosophique d'Althusser ne s'épuise pas dans les effets critiques constatés dans son premier contexte d'inscription. Cette aptitude à lire une conjoncture, par des différenciations qui vont à rebours d'une inclusivité, dans l'Europe de l'après-guerre ou en ce moment de " capitalisme tardif », dégage des contradictions réellement existantes, non-solubles dans une communauté sentimentale. Cela fait du «projet althussérien » un opérateur pratiquant l'interrogation de l'histoire sous l'angle d'une incomplétude tragique. C'est en tout cas ce que laisse entendre la contribution de Panagiotis Sotiris, "From the "Hidden God" to the Materialism of the Encounter : Althusser and Pascal» (p. 152-167). Dans le texte de 1946, au lieu de la communion des «bons sentiments », se dresse la figure du prolétariat, agent d'une histoire effectivement conflictuelle.

4 La première des contributions, « Althusser and Religion » (p. 7-17), est de Stanislas Breton (1993). Il s'agit de la traduction d'un texte qui figure dans le volume Althusser Philosophe, sous la direction de Pierre Raymond (1997). Hamza avait conclu son introduction en posant comme prémisse, en une variation sur un aphorisme de Lénine sur la relation entre le Capital de Marx et la Logique d'Hegel: «It is impossible to completely understand Althusser's philosophy without having thoroughly studied and understood his Early Catholic Writings » (p. 6). D'où, en toute cohérence, le début du volume avec le texte de Stanislas Breton, qui relève que la dette d'Althusser vis-à-vis de son milieu chrétien s'exprime en exigences de rigueur : «Dans son éloignement, il restait conscient de ce qu'il devait au milieu chrétien dont il avait aimé certaines exigences de rigueur tant du point de vue 
de la conduite de l'action que de la structure de la pensée " (Raymond, 1997, p. 162). (Si nous citons le texte de Stanislas Breton en sa version d'origine, c'est que le raidissement de la syntaxe du texte anglais ne rend justice ni à Breton ni aux textes d'Althusser de la période 1945-1951.)

5 Le texte de Stanislas Breton valide l'hypothèse intuitive d'une continuité du projet philosophique, par-delà les ruptures ou les coupures. Or si Hamza, dans son introduction, note qu'Althusser avoue avoir perdu la foi en 1947, il précise aussitôt : "But this is not important for our volume» (p. 5). En effet la thèse principale du présent volume, nous indique-t-il, porte plutôt sur la religion: «it was his Catholicism (i.e. Christianity) that was the main reason why Althusser became a communist philosopher » (p. 5). Le titre du volume mentionne donc les termes "Religion » et "Theology », jouant, à l'occasion, d'une opposition entre les deux termes, qu'unit cependant leur référence sous-jacente à la structure comme condition de formulation critique et d'intervention pratique, en antithèse à la certitude intuitive qu'est la foi. La contribution de Breton conforte cette approche, axée sur la formation institutionnelle et discursive qu'est le christianisme, notamment en sa version catholique, qui en contrepartie tend à minorer la force de justification qui est le propre de la foi, en corrélation aussi à une minoration de la subjectivité. Breton conclut ainsi : «En tant que distincte de la religion, la foi ellemême ne serait donc pas la solution de rechange sur la voie qui l'avait éloigné de ses amours d'antan. En dépit de sa relative radicalité, elle n'en continuait pas moins de se mouvoir dans le cercle enchanté qui lie, d'une solidarité infrangible, la théologie et la téléologie, l'ontologie et l'idéologie » (Raymond, 1997, p. 165).

6 Il émerge du témoignage et acte de compréhension philosophique de Stanislas Breton le profil d'un Althusser rétif au fidéisme et à la subjectivité, formé à la discipline d'une «structure de la pensée ». Celle-ci s'entend comme une régulation du discours qui rompt avec la complaisance affective ou subjective, avec l'hypothèse d'un en-commun «des sentiments». Au lieu d'un fidéisme, un institutionnalisme; une fidélité maintenue vis-à-vis de la rigueur des formations et des discours non-charismatiques. Engagement et ratio, au lieu d'un fidéisme auto-suffisant. La continuité, après l'éloignement de l'Église, serait à chercher dans le constat de la nécessité d'une organisation et de l'insuffisance de la sola fides. Plus loin, dans l'article de Knox, "Althusser's Spinozism and the Problem of Theology» (p. 101-114), il sera de nouveau question de la pertinence ou non d'une perspective sur "Louis Althusser avant Althusser" ou sur le marxisme d'Althusser qui serait dérivé d'une approche philosophique issue du versant subjectif et romantique du hégélianisme, passant par le jeune Marx, plusieurs fois ressuscitée comme le fondement de ce que Knox qualifie de "sorte d'ontologie morale qui met en exergue l'aliénation et la rédemption » (p. 112) plutôt que le fait primaire de l'exploitation. À propos de Roland Boer, auteur de Marxism and Theology, cinq tomes (2009-2014) et dans le présent volume de l'article "Althusser's Religious Revolution» (p. 18-30), Knox constate: "The main virtue of Boer's reading of Althusser is to distinguish between theology as a set of views or positions on the nature of reality, that is, a doctrine, and ecclesiology, which concerns the nature of religious institutions and their history, in a word: the Church» (p. 104). Il poursuit par le constat suivant: «Boer's hermeneutic is decidedly phenomenological and its ontological implication is expressive » (p. 105). En revanche, pour Knox, "the catholicity of Althusser's concept of ideology is deemed most intelligible if we regard it as the expression of a persistent ecclesiology in his thought » (p. 105-106). L'ajustement à la rigueur de l'institution ecclésiologique se laisserait-il convertir en discipline institutionnelle de l'appareil du parti ? C'est en tout 
cas l'une des options permettant une mise en ordre du parcours philosophique et de ses césures, si on veut privilégier l'hypothèse de la continuité. À ces deux moments du parcours, avant 1951 et après, se trouverait ainsi maittrisée, par la critique en raison, la propension à l'affect narcissique. Du coup, se trouveraient aussi mises à distance les voies de la dialectique des Lumières, où inextricablement se débattent Adorno et Horkheimer, dans les impasses de l'authenticité et de son contraire. Pour Knox, toute tentative de saisir le projet philosophique d'Althusser, ses continuités ou ses ruptures, sera inopérante si la compréhension est tentée selon le schéma vitaliste ou téléologique d'une continuité faisant sens, où l'analyste va relever les actes et les dires qui jalonnent le parcours comme autant de signes lisibles selon un postulat d'expressivité. Une telle approche serait étrangère à la structure de la pensée et d'action qui est celle d'Althusser. Ainsi inscrit-il le parcours d'Althusser sous l'austère règle d'une inexpressivité délibérée, en rupture avec le postulat d'une continuité expressive qui ne peut que «faire sens » pour l'analyste du parcours, scrutant les méandres des moments et positions successifs.

7 Sur ce point de la non-pertinence d'approches souscrivant au postulat d'une continuité expressive, Knox est convaincant, jusque dans son constat de la "non-rencontre " entre Althusser et la conjoncture de mai 68, peut-être l'ultime accomplissement d'une expressivité performative unanimiste, débouchant sur une internationale des bons sentiments : "When May 1968 broke out, Althusser's theoreticism suddenly seemed wide of the mark, if not irrelevant. On the Reproduction of Capitalism was his response to his situation. " (p. 108)

8 Interrogation de l'articulation chez Althusser entre théologie et religion, politique et philosophie, le livre ne cherche pas à restituer en épaisseur une histoire culturelle des milieux catholiques de l'après-guerre. À cet égard, l'ensemble du volume, par sa focalisation stricte sur les enjeux de philosophie et de théologie manque de cet esprit de finesse mise en évidence dans le texte de Stanislas Breton. Aucune référence dans le livre aux théologiens de la période d'avant le concile de Vatican II ; aucune référence non plus à Gilson ou Maritain. Pourtant «L'internationale des bons sentiments » ou "Une question de faits» (1949) attestent que, pour Althusser, l'explication avec la conjoncture appelle des procédures de compréhension à la fois empathiques et informées, en préalable à la critique. Le texte de 1949 distingue clairement entre les compromissions de l'Église avec des gouvernements fascistes, la collusion avec des gouvernements profondément réactionnaires (Irlande, Canada, Amérique du Sud), la voie moyenne de la démocratie chrétienne, avant d'envisager les «Perspectives d'une réduction pratique " (Althusser 1994, p. 272), hypothèse par laquelle Althusser entend que "la "réduction théorique" de l'inquiétude religieuse présente nous a conduit à reconnaître dans l'aliénation religieuse l'origine même de l'inquiétude. Il faut envisager alors les moyens qui permettent de "réduire" pratiquement cette origine, en la détruisant pour la convertir dans sa vérité » (ibid., p. 272-273).

Même en tenant compte de la centralité dans ce livre de l'articulation chez Althusser entre la théologie et le "projet philosophique ", la règle posée par Balibar, d'un projet philosophique fortement tributaire de son contexte spécifique, aurait dû motiver une attention plus précise aux conjonctures. La justesse des textes de 1946 est fonction de l'articulation entre la généralité critique et la prise en compte spécifique des faits de la conjoncture. En l'absence d'une attention suffisamment précise au texte d'Althusser ou à la conjoncture abordée, l'intention déclarée dans certains textes de pratiquer la 
critique d'une conjoncture actuelle peut échouer, à cause du côté forcé de l'attelage proposé, entre la conjoncture examinée et un discours qui se prétend éclairé par le schéma critique althussérien. Cela est indiscutablement le cas, s'agissant de la contribution d'Isa Blum, «Battles of Nostalgic Proportion: The Transformations of Islam-asHistorical- Force in the Ideological Matrix of a Self-Affirming "West" ", où la conjoncture se trouve trop simplement allégorisée, réduite à un théâtre d'ombres (p. 182-197). Or ce que laissait entendre la proposition de Balibar, c'est que le projet althussérien produit ses effets en articulation avec un contexte spécifique. Il paraît évident que le projet garde ses propriétés de pratique critique en d'autres conjonctures. Mais cela n'en fait pas un schéma général et passe-partout, mobilisable sans discernement ou précision critique.

10 C'est dans le chapitre rédigé par Roland Boer, "Althusser's Religious Revolution » (p. 18-30), que nous trouvons une exégèse attentive du texte " Une question de faits " (p.18). L'exégèse cherche à répondre à la question que pose Boer, déjà abordée par Stanislas Breton dans ses entretiens avec Althusser «pendant les dures années d'une décennie, de 1980 à 1986 en particulier » (Raymond, p. 163) : «Is it possible that Althusser was for a time a theologian of liberation, indeed that he may be counted among those who form part of the tradition of revolution Christianity? His distinct contribution-albeit not without some problems-is to argue that a revolution in personal religious life is analogous to a socialist revolution » (p. 18). Le nœud de la question que pose Boer est en germe dans le titre de sa contribution : «Religious Revolution». Révolution de la religion ou dans la religion? Ou relève de la religion? L'interrogation ne fait que répéter l'interrogation formulée, dès les Évangiles et les Épitres de Paul, quant à un ordre institutionnel réellement existant de la religion ou quant à l'accomplissement ou abolition de celle-ci. Boer met en exergue la singularité de ce premier Althusser. Il revient donc à ce passage dans le texte de 1949 où il est question des "Perspectives d'une réduction pratique ": "For Marx, truth entails a socialist revolution so that a new mode of production may be constructed in which alienation is no longer a reality. Given that alienation is the cause of religion, religion itself will disappear. For Althusser, the truth sought is quite different, for it is nothing less that the recovery of religious truth and authentic commitment» (p. 25). Boer relève la proposition d'Althusser portant sur les conditions d'une double émancipation : "Althusser writes: 'the reduction of collective religious alienation presuppose this political and social struggle as the condition without which no emancipation, not even religious emancipation, is conceivable' ». Althusser se placerait ainsi dans un double territoire, à la fois sur le terrain des luttes sociales, qui est le propre du Marxisme, et sur une autre scène, ailleurs ou en retrait, où une émancipation religieuse est concevable. C'est à cette condition, selon Boer, qu'Althusser se situe ailleurs que dans l'habituel territoire marxiste. Et Boer de conclure, à propos de cette dualité du projet d'émancipation dans "Une question de faits »: « He does not wish to abolish the church, but to save it » (p. 25).

11 On pourrait objecter à cela que la protestation d'une fidélité vis-à-vis de ses « previous loves » (p. 16), ce que Breton a appelé, s'agissant d'Althusser, ses « amours d'antan », est un passage obligé dans tout geste de détachement ou de congédiement. Et si nous revenons à l'hypothèse de Balibar concernant l'inscription des écrits d'Althusser dans la conjoncture où ils produisent leurs effets, si « Une question de faits » s'adresse à un contexte social, à la situation des Églises catholiques dans les sociétés qui, à des degrés divers, ont été éprouvées et transformées par les années de guerre, le texte s'adresse aussi à son auteur, Louis Althusser, sujet ou agent d'une « émancipation religieuse » en 
cours, qui peut s'entendre comme le dépassement ou abandon ou l'accomplissement des données effectives (les faits réels) d'un engagement catholique antérieur. Selon une première lecture possible, le texte de 1949 s'entend comme un travail de deuil où l'auteur s'acquitte d'une dette. Boer semble vouloir sauver ce qui y a de proprement religieux dans l'Althusser de la maturité politique marxiste, et établir une ligne de vie souterraine reliant ce premier Althusser et les théologies de la libération. Cela suppose une articulation entre la révolution dans les conditions sociales effectives et une révolution intérieure : une métanoia. On peut se demander si cette lecture est ajustée à la trajectoire ultérieure d'Althusser, à ses interventions dans d'autres conjonctures et à sa pratique de la théorie. Boer, à son tour, écrit dans une autre conjoncture : à la fois post-soviétique, en aval du communisme réellement existant; en aval, aussi, des socialdémocraties réformistes de la période 1974-2008. Une telle séquence de coupures et d'effondrements rend plausible le geste de Boer consistant à retrouver un potentiel intact de force théorique, dans ce texte qui, lors de sa publication, fait partie, avec "Lettre à Jean Lacroix ", d'un avis d'éloignement. Le geste de Boer, consistant à retrouver, en vue de son réinvestissement dans la conjoncture actuelle, une force critique propre au "Louis Althusser avant Althusser ", peut s'entendre comme un geste de reprise religieuse de ou sur Althusser, comme un geste qui à son tour est lisible comme le symptôme du profond désarroi actuel, pointé par Tsoneva, à propos de la résonance que reprend "L'internationale des bons sentiments » en un temps de crise politique et environnementale.

Le texte de Boer échappera-t-il à la rigueur clinique dont fait preuve Althusser dans son texte de 1946? En effet, la suite des chapitres de ce livre évalue différentes interventions théoriques dans une conjoncture plus récente, qui en grande partie est "post-althussérienne ». Les deux figures de la scène philosophique les plus couramment évoquées dans les contributions sont Žižek et Badiou : notamment par Gabriel Tupinambá dans « Splitting Althusser at the Point of Religion» (p. 64-88). Ces deux penseurs reprendraient une partie, ou l'un des côtés, d'une impossible unité postulée par Althusser : "Each philosopher develops one side of the impossible unity postulated by Althusser under the name of "Marxist dialectic", a choice most clearly visible in the way each of them mobilises the ontological basis of real abstractions in their disparate reading of the Christian event» (p. 84). Dans la suite du volume, Ted Stolze évoque le retour à Paul, dans la conjoncture qui est la nôtre: "Paul of Tarsus, Thinker of the Conjuncture " (p.129-151). On notera aussi la contribution de Vittorio Morfino, «Eschatology à la Cantonade : Althusser beyond Derrida ( (p. 115-128), évaluation, plutôt sévère, du geste de Derrida de vouloir faire entendre la tonalité d'un marxisme messianique de l'après-coup et d'un temps "out of joint ». Morfino trouve plus de précision et d'ajustement conjoncturel dans la mise en scène par Giorgio Strehler d'une pièce de Carlo Bertolazzi, El Nost Milan (1962), à laquelle Althusser consacra un article.

On revient à des enjeux et impasses que le parcours philosophique d'Althusser, tel qu'il est interrogé dans ce livre, nous oblige à penser : la question de l'inscription d'un acte de critique dans une conjoncture; les épuisements successifs, ainsi que les régénérescences possibles, des interventions théoriques pratiquées dans un parcours philosophique; la tentation ou l'espérance de faire émerger une communauté ou une "internationale", qu'unit "les bons sentiments" ou un désarroi en partage; l'invocation de l'événement comme force de rupture, par Badiou ou Žižek; la production ou réception d'un projet philosophique dans l'horizon d'attente d'une cohérence «expressive ». L'ensemble de ces traits désigne une conjoncture et une 
scène philosophique en aval d'Althusser. Dans le témoignage de Stanislas Breton, ou dans les autres contributions, notamment celle de Panagiotis Sotiris, le dernier moment de ce parcours philosophique, celui du "matérialisme aléatoire», donc d'une contingence radicale, est interrogé dans sa relation aux moments antérieurs. Le livre témoigne ainsi d'un intérêt renouvelé pour "Louis Althusser avant Althusser », pour l'hypothèse d'une «révolution religieuse» qui s'entend comme relève et accomplissement de ce moment religieux, à travers un événement qui serait de l'ordre d'une "émancipation religieuse». Impossible de dire si un tel "retour à Louis Althusser » serait fidèle ou non au parcours philosophique d'Althusser. Dans l'horizon ouvert du matérialisme aléatoire, cela relève à la fois de l'inattendu et du possible. 